\title{
Baudelaire et la parole déchue
}

\section{Baudelaire and the fallen poetry}

Eduardo Horta Nassif Veras

Universidade Estadual de Campinas, Campinas, São Paulo / Brasil eduardohnveras@gmail.com

Résumé : Cet article a pour but de présenter une analyse de la place de la théologie dans la poétique de Charles Baudelaire. Après la mise au point des études les plus récentes qui mettent l'accent sur la révision critique des rapports du poète des Fleurs du mal à la modernité poétique et sur la présence des thèmes dits antimodernes dans son œuvre, on se propose de montrer que la poésie baudelairienne se construit autour d'une conception déchue de la vie et de la parole poétique.

Mots-clés : Baudelaire ; antimodernité ; péché originel ; théologie.

Abstract : This article aims to present an analysis of the place of theology in Baudelaire's poetics. After an analysis of the most recent studies focusing on critical reviews of the relations of the poet of Fleurs du mal with the poetical modernity, as well as the presence of themes considered antimodernist in his work, I propose to show that Baudelaire's poetry is constructed based on a conception of life and of a poetical discourse based on the fall.

Keywords : Baudelaire; antimodernity; original sin; theology.

Recebido em: 29 de abril de 2017.

Aprovado em: 7 de junho de 2017. 


\section{Une révision critique}

Un bref regard sur le panorama actuel des études baudelairiennes en France suffit pour dévoiler ce que l'on pourrait appeler une révision critique des rapports de Baudelaire à la modernité. Mise en œuvre principalement dans la critique universitaire, cette révision propose une interprétation de la poésie et de la pensée de Baudelaire de plus en plus éloignée de celle qui voyait dans l'auteur des Fleurs du mal le " poète de la modernité » tout simplement. Les récentes parutions de la dernière étude d'Antoine Compagnon, Baudelaire : l'irréductible (2014), et du recueil d'essais d'Yves Bonnefoy intitulé Le Siècle de Baudelaire (2014) en témoignent parfaitement. L'analyse des dernières publications en France met en évidence surtout les efforts de déconstruction du mythe du poète de la modernité tout en révélant le nouvel intérêt que la critique porte aujourd'hui sur le "dernier Baudelaire », celui des fragments posthumes, du « Peintre de la vie moderne » et des poèmes en prose. Deux travaux directement attachés au «Centre de Recherches sur la Littérature Française du XIXe siècle » de la Sorbonne, un des principaux foyers de ce mouvement révisionniste, peuvent témoigner de cet intérêt croissant : le recueil d'articles intitulé Lire Le Spleen de Paris de Baudelaire (2014), dirigé par André Guyaux et Henri Scepi, et la Bibliographie du Spleen de Paris (1855-2014) (2015), réunie et préfacée par Andrea Schellino.

Le rapport de Baudelaire à la théologie constitue l'un des principaux axes de cette révision critique et continue d'attirer l'intérêt de nombreux spécialistes. L'une des principales raisons de cet intérêt est le fait que le dialogue avec la théologie représente la mise en question du projet moderne d'autonomie esthétique auquel Baudelaire fut à plusieurs reprises associé. Datant principalement des deux derniers décennies, les relectures de la modernité baudelairienne veulent montrer par exemple que le poète des Fleurs du mal et du Spleen de Paris se dresse souvent contre la « folie de l'art », contre le « goût immodéré de la forme » (BAUDELAIRE, 1976, v. 2, p. 48-49), et que cette critique de l'absolu esthétique vient souvent accompagnée de nombreuses manifestations de teneur éthique et religieuse, principalement dans le cadre des poèmes en prose. C'est bien l'un des principaux arguments du travail d'Edward Kaplan, Baudelaire et Le Spleen de Paris : l'esthétique, l'éthique et le religieux (2015), finalement traduit en français vingt cinq ans après sa parution en anglais. Très attentif aux 
subtilités de l'ironie baudelairienne, le chercheur américain essaie de montrer que la poésie de Baudelaire se caractérise par le double mouvement de jouissance et de dénonciation du «blasphème de l'art » (BAUDELAIRE, 1975, v. 1, p. 23). Cette dernière expression, tirée du poème en vers Le Masque, rappelle encore une fois la question religieuse, sur laquelle je me propose de réfléchir par la suite.

En tête de toutes les références de Baudelaire à la théologie on retrouve la doctrine du péché originel. Le recours à ce dogme représente une sorte de contre-discours au moyen duquel le poète met en cause la philosophie du progrès et le projet moderne d'autonomie esthétique et morale - associées respectivement à l'absolu romantique de l'art comme forme supérieure de la connaissance et à la théorie rousseauiste du bon sauvage. La « théorie » baudelairienne de la « vraie civilisation », lit-on dans le fragment XXXII de Mon Cour mis à nu, « n'est pas dans le gaz, ni dans la vapeur, ni dans les tables tournantes, elle est dans la diminution des traces du péché originel » (BAUDELAIRE, 1975, v. 1, p. 697). L'idée du péché originel s'oppose à ce que Baudelaire appelle la « fatuité moderne » (BAUDELAIRE, 1976, v. 2, p. 580). Héritage augustinien, ce concept occupait déjà une place centrale dans la pensée de Joseph de Maistre, dont l'influence sur la pensée baudelairienne est avouée dans le fragment II d'Hygiène : « De Maistre et Edgard Poe m'ont appris à raisonner » (BAUDELAIRE, 1975, v. 1, p. 669). Selon le théologien français, « [...] il n'y a rien de si attesté, rien de si universellement cru sous une forme ou sous une autre, rien enfin de si intrinsèquement plausible que la théorie du péché originel » (DE MAISTRE, 2007, p. 189). Bien plus qu'un parti pris théorique, néanmoins, le recours de Baudelaire à ce concept théologique correspond à des procédures poétiques remarquables, dont la déconstruction ironique de l'ivresse poétique, la dénonciation de l'illusion romantique et les efforts de conciliation entre la poésie et la prose.

Les influences que la pensée antimoderne de Joseph de Maistre a exercées sur Baudelaire dépassent le niveau de l'adhésion idéologique du poète à la doctrine du péché originel et à l'anthropologie catholique en générale. Mon hypothèse est que la poétique baudelairienne se construit de manière analogue à la pensée maistrienne en dramatisant par le moyen du langage poétique l'ambiguïté de l'homme déchu, « de l'homme créé bon et devenu mauvais » (RICEUR, 2009, p. 460). Attachée à cette « anthropologie de l'ambiguïté » (RICCEUR, 2009, p. 460), dont parle Paul Ricœur dans son exégèse du mythe biblique de la chute, la poétique 
de Baudelaire oscillera entre la parole esthétique et la parole éthique, entre l'extase et l'horreur de la vie (BAUDELAIRE, 1975, v.1,p. 703) et de l'art lui-même. La poétique baudelairienne se tiendrait donc en suspension entre les pôles contraires de l'extase blasphématoire des images (l'ivresse de l'art), d'un côté, et l'effort critique de déconstruction du langage romantique des correspondances, celles-ci associables à la quête de l'unité perdue, au « désir d'infinitude », (RICEUR, 2009, p. 467) de l'autre.

Aux influences de Joseph de Maistre sur la pensée de Baudelaire il faut ajouter sans doute celles de Pascal. L'héritage pascalien de cette « anthropologie de l'ambiguiité » est attesté par l'étude comparative Pascal et Baudelaire (2011), de Jean Dubray. D'après Pascal les contradictions de l'homme trouvent aussi leur source dans une « nature humaine blessée intimement par la chute originelle » (DUBRAY, 2011, p. 24). On connait déjà suffisamment la place que ce concept de péché originel tient dans la pensée de Baudelaire et l'importance qu'il prend, « sous l'influence de Joseph de Maistre » (DUBRAY, 2011, p. 24), dans les derniers écrits du poète. Il faut maintenant se demander sur les points de contact entre cette vision antimoderne de la vie et la structure interne de la poétique baudelairienne. Pour autant il faudra montrer que la poésie de Baudelaire se tient, comme l'homme déchu, à la frontière du désir d'infinitude, qui rappelle son origine divine, et du besoin de séparation, qui est à la base de la liberté et de la responsabilité éthique. En d'autres mots, je tenterai de montrer que la poésie de Baudelaire oscille paradoxalement entre l'éloge et le refus de la poésie elle-même en tant que langage capable de toucher l'origine des choses.'

Il est intéressant de remarquer que ce mouvement d'hésitation critique entre deux catégories linguistiques, à savoir celle des correspondances, où tout se confond " dans une ténébreuse et profonde unité » (BAUDELAIRE, 1975,v. 1, p. 11), et celle de la parole philosophique, antidote contre la " passion frénétique de l'art» (BAUDELAIRE, 1976, v. 2, p. 48), n'est pas très éloigné du scepticisme pascalien « à l'égard des deux principes de vérité qui sont les sens et la raison » (DUBRAY, 2011, p. 24). On retrouve ici la posture critique qui caractérise la pensée et la poésie de Baudelaire. Tout en refusant toute

\footnotetext{
${ }^{1}$ Selon Paul Ricœur (2009, p. 216), « [...] le symbole poétique nous montre l'expressivité à l'état naissant ; dans la poésie le symbole est surpris au moment où il est un surgissement du langage. »
} 
forme d'absolutisation, le poète se confond avec le critique ${ }^{2}$ en produisant une poésie qui met toujours en cause son propre statut d'art sans se confondre pour autant avec le langage de la philosophie, chacun, l'art et la philosophie, jouant le rôle de limiter le désir d'absolu de l'un et de l'autre : « la folie de l'art est égale à l'abus de l'esprit. ${ }^{3}$ La création d'une de ces deux suprématies engendre la sottise, la dureté du cœur et une immensité d'orgueil et d'égoïsme » (BAUDELAIRE, 1976, v. 2, p. 49).

Fonctionnant comme une forme de résistance antimoderne contre « l'hubris sacrilège des modernes »(COMPAGNON, 2005, p. 90), le péché originel est évoqué par Baudelaire dans sa critique de la conception moderne du beau :

La plupart des erreurs relatives au beau naissent de la fausse conception du XVIIIe siècle relative à la morale. La nature fut prise dans ce temps-là comme base, source et type de tout bien et de tout beau possible. La négation du péché originel ne fut pas pour peu de chose dans l'aveuglement général de cette époque (BAUDELAIRE, 1976, v. 2, p. 715).

Le beau baudelairien n'est donc pas séparable de la condition déchue de l'homme, parce que «la beauté date du péché ; elle est inséparable de la mélancolie ; elle est satanique »(COMPAGNON, 2005, p. 89). Symbole de la séduction et de la déperdition, le Démon apparaît plusieurs fois associé à l'art, comme dans ces vers de «La Destruction» : « Parfois il [le Démon] prend, sachant mon grand amour de l'Art, / La forme de la plus séduisante des femmes » (BAUDELAIRE, 1975, v. 1, p. 109). L'association entre ces trois éléments - le Démon, l'Art et la

\footnotetext{
${ }^{2}$ À propos des rapports entre le poète et le critique, Baudelaire écrit dans son essai sur Richard Wagner : «Ce serait un événement tout nouveau dans l'histoire des arts qu'un critique se faisant poète, un renversement de toutes les lois psychiques, une monstruosité ; au contraire, tous les grands poètes deviennent naturellement, fatalement, critiques. Je plains les poètes que guide le seul instinct; je les crois incomplets ». (BAUDELAIRE, 1976, v. 2, p. 793).

${ }^{3}$ D'une certaine façon Baudelaire y met en œuvre une caractéristique de la poésie en générale. Selon Michel Deguy, le logos poétique se placerait dans l'espace de l'entre, il refuserait aussi bien l'absolu de la confusion que celui de la séparation radicale : " Il s'agit "à la fin" de veiller sur la différence - appelons-la tantôt dedans-dehors - en démentant (aussi par le rire) les assimilations imprudentes, les identifications intolérantes et meurtrières, et les exclusions tranchantes jusqu'au sang. » (DEGUY, 1987, p. 23).
} 
femme - aboutit, dans le même poème, sur l'expérience de la confusion et de la destruction, en d'autres termes, sur le symbole cosmique du chaos.

Les thèmes de la destruction et du chaos se lient à celui du sacrifice, dont le concept se présente comme une autre marque de l'influence maistrienne sur la pensée de Baudelaire. La malédiction humaine étant un fait incontestable, le mal étant définitivement attaché à la vie, Joseph de Maistre voit dans le sacrifice un moyen d'expiation des coupables. Le « salut par le sang » (DE MAISTRE, 2007, p. 839) établit une sorte de dialectique du chaos et de l'ordre, de la destruction et de la régénération (ne se confondant pas avec la rédemption cela va sans dire) qui se répète dans la poésie de Baudelaire, non seulement au niveau thématique mais aussi dans l'immolation de la conscience immergée dans l'absolu blasphématoire de l'art. Le martyre du poète est marqué par l'ambiguïté du plaisir et de la douleur, de l'extase et de l'horreur qui est bien celle qui définit l'homme déchu, capable en même temps de jouir et de regretter son propre mal.

La révolution mise en œuvre spécialement par le « dernier Baudelaire » est moins celle représentée seulement par la découverte de l'illusion de la figuration poétique, dont parle Barbara Johnson (1979, p. 65), mais plutôt celle qui met en évidence le lieu hypercritique de la poésie, caractérisé par un double refus, celui de la « folie de l'art» (BAUDELAIRE, 1976, v. 2, p. 48) et celui de «l'art philosophique » (BAUDELAIRE, 1976, v. 2, p. 598). Cela veut dire que cette poésie-critique a pour cible aussi la propre puissance critique du scepticisme moderne. Encore une fois, la dramatisation poétique de la méchanceté originelle dépasse le niveau des références au concept de péché originel tout en mettant en place un langage caractérisé, lui aussi, par l'amalgame des deux principes de vérité, le sens et la raison, du corps et de l'esprit, sans prendre le parti ni de l'un ni de l'autre. Parce qu'il n'y a pas de poésie sans enchantement chez Baudelaire, le travail de l'artiste coïncide alors avec une sorte de bataille contre la Nature, bataille que paradoxalement il ne pourra jamais vaincre complètement sous peine de vider le langage de tout contenu poétique. C'est pourquoi l'expérience du beau est inséparable de la souffrance :

Ah ! faut-il éternellement souffrir, ou fuir éternellement le beau? Nature, enchanteresse sans pitié, rivale toujours victorieuse, laisse-moi ! Cesse de tenter mes désirs et mon orgueil ! L'étude du beau est un duel où l'artiste crie de frayeur avant d'être vaincu (BAUDELAIRE, 1975, v. 1, p. 278-279). 


\section{Le plein de la poésie}

L'ironie baudelairienne n'aboutit jamais sur le néant. D'abord parce que la déconstruction des « illusions » ne représente qu'un des deux piliers de l'expérience poétique de Baudelaire, qui dans un fragment de Fusées affirme qu'il y a « deux qualités littéraires fondamentales : [1e] surnaturalisme et [1'] ironie » (BAUDELAIRE, 1975, v.1, p. 658). Ce qu'il appelle surnaturalisme s'attache à la puissance de la parole poétique, à quelque chose qui dépasse le niveau de la littéralité, de la matérialité du langage tout simplement. Parce qu'il y a « dans le mot, dans le verbe, quelque chose de sacré, qui nous défend d'en faire un jeu de hasard » (BAUDELAIRE, 1976, v. 2, p. 118), la poésie ne se réduit pas à la manipulation du signifiant, au travail de la forme. C'est aussi l'avis d'Yves Bonnefoy qui, dans son dernier recueil d'essais, n'hésite pas à mettre à plusieurs reprises l'accent sur l'hiatus qui sépare Baudelaire de la poésie du XXe siècle, plus attachée, selon lui, à l'héritage de Mallarmé. Son insistance sur ce point n'est pas sans rapport avec la question de l'ambivalence de la parole poétique baudelairienne dont nous nous occupons ici :

Le XIXe siècle en poésie n'a pas eu beaucoup d'héritiers dans le XXe. Il est même frappant de constater que, dressant de plus en plus fortement Mallarmé contre Baudelaire, reprenant à frais nouveau l'antique dessein de la rhétorique, la littérature, cet antique déni de la poésie, a, réserve faite du surréalisme et de Proust, bien clairement compris que c'était le mot son péril : d'où une immense manœuvre dans les réseaux de laquelle l'heure présente est en risque d'étouffement. Cette manœuvre ? Décider que le mot, qui paraît comme tel dans le travail de la forme sur la parole, doit être mis en rapport, non plus avec ce qu'il nomme dans l'au-dehors du langage, mais avec tous les autres mots qui sont dans la langue (BONNEFOY, 2014, p. 15).

Bonnefoy entend que la poésie du XXe siècle se caractérise, à l'instar de l'héritage mallarméen, par le refus du mot en tant que phénomène incarné dans l'histoire, c'est-à-dire de la parole poétique comprise comme manifestation de la condition de l'homme dans le monde. Les arguments de Bonnefoy ne sont pas sans rapport à la question de la « banalisation de l'incroyance » (BONNEFOY, 2014, p. 7) et de ses conséquences sur la production poétique. Baudelaire 
aurait été le premier poète à concevoir le «plein de la poésie » dans un monde de plus en plus rongé par le vide métaphysique. La découverte baudelairienne de l'autonomie de la poésie en tant que langage n'entraine pas le renfermement de la parole poétique sur elle-même, mais le « débat entre soi et soi », « entre, dirais-je, le moi bâti par la pensé conceptuelle et le Je qui se souvient de l'unité qu'elle occulte et même censure " (BONNEFOY, 2014, p. 10). En d'autres mots, la découverte du «plein de la poésie » est à la base du conflit baudelairien entre l'image et le concept, entre l'unité des correspondances et la prise de distance ironique. L'idée de Bonnefoy est que le « retrait de la religion » associé à la révélation de la « poétique en sa différence » (BONNEFOY, 2014, p. 12) aurait permis à Baudelaire de rejoindre la finitude et l'être. Celui-ci est identifié aux souvenirs de l'enfance, des années profondes, dont les restes de la « présence immédiate » peuvent être perçus dans les « mots les plus simples de l'exister quotidien » (BONNEFOY, 2014, p. 14).

La lecture de Bonnefoy nous permet de comprendre d'abord que la poésie de Baudelaire ne se détache pas de la vie, des souvenirs et de la reconnaissance de la finitude. Bien qu'elle soit capable de reconnaître et même d'inaugurer le « plein de la poésie », cette poésie paraît s'arrêter là où les poétiques modernes trouvent leur point de départ. De cette manière, Baudelaire reste attaché au XIXe siècle tout en faisant un pas en arrière par rapport à l'autonomie esthétique qui définit les poétiques modernes. Cela nous permet de dire toujours avec Bonnefoy que la poésie se caractérise, chez Baudelaire, comme une sorte de " contestation de l'illusion esthétique » (BONNEFOY, 2014, p. 90), c'est-à-dire la mise en question autocritique de la parole poétique.

Mais Bonnefoy trouve la source de cette présence antérieure à tout langage dans la biographie de Baudelaire, dans ses souvenirs d'enfance, dans l'évocation poétique de ses expériences amoureuses. Il nous paraît, toutefois, que les rapports entre la parole et la vie se trouvent ailleurs, dans la conception baudelairienne de l'homme, dans son « anthropologie de l'ambiguiité », dans ses héritages pascaliens et surtout maistriens. Plutôt que dans les textes critiques, où il revient à plusieurs reprises au concept chrétien de péché originel, dans les poèmes des Fleurs du mal Baudelaire met en scène le personnage d'un poète toujours caractérisé par une duplicité semblable à celle qui définit l'homme déchu : «Lorsque, par un décret des puissances suprêmes, / Le Poète apparaît en ce monde ennuyé » (BAUDELAIRE, 1975, v. 1, p. 7). 
Figure intermédiaire entre le divin et l'humain, ce Poète est condamné à la souffrance dès le débout de son parcours dans Les Fleurs du mal. C'est bien une sorte d'exil dans son propre monde, tel celui des « vastes oiseaux des mers » (BAUDELAIRE, 1975, v. 1, p. 9-10) :

Le Poète est semblable au prince des nuées

Qui hante la tempête et se rit de l'archer;

Exilé sur le sol au milieu des huées,

Ses ailes de géant l'empêchent de marcher.

Qu'est-ce que le « divin » sinon ce que le poète lui-même appelle le « surnaturalisme », ce qui dépasse le monde naturel où chaque chose a sa place et son identité ? Et qu'est-ce que «l'humain » sinon ce qu'il appelle l'ironie, ce qui s'oppose à l'unité idéal de Dieu ? D'un côté, il y a le souvenir de l'unité perdue, à laquelle tout poète est capable d'accéder, selon ce que l'on peut lire dans l'article sur Banville : « tout poète lyrique, en vertu de sa nature, opère fatalement un retour vers l'Eden perdu » (BAUDELAIRE, 1976, v. 2, p. 165); et de l'autre, la « vorace Ironie / qui me secoue et mord » (BAUDELAIRE, 1975, v. 1, p. 78). Ce sont là les deux côtés du sacré : l'unité divine et la séparation satanique, le paradis et la chute, l'innocence et le mal. Ces deux espaces étouffants définissent de manière claire l'anthropologie baudelairienne de l'ambiguïté : « il y a dans tout homme, à toute heure, deux postulations simultanées, l'une vers Dieu, l'autre vers Satan. » (BAUDELAIRE, 1975, v. 1, p. 682). L'homme, ou plutôt l'homme-poète, se trouve, donc, dans le simultané, paradoxalement plongé aussi bien dans l'unité de Dieu que dans le doute diabolique. Or, le Poète (ce personnage des Fleurs du mal) veut bien être l'homme doué de « conscience dans le mal» (BAUDELAIRE, 1975, v. 1, p. 80), c'està-dire celui qui est capable de jouer sur les deux postulations, ce qui lui permet de se tenir en suspension, hors de la portée de toute forme d'absolu.

Il ne s'agit plus d'une dispute entre le bien et le mal, comme dans la mythologie catholique, cela va sans dire, mais plutôt entre deux types également étouffants d'absolus. Entre les correspondances, qui postulent l'unité entre le langage et le monde, et la critique, qui postule le contraire et creuse le vide. Baudelaire, à la différence des principales poétiques modernes, ne choisit ni les unes ni l'autre, mais plutôt l'espace 
mouvant du paradoxe voire de l'oxymore. ${ }^{4}$ Espace qui se creuse toujours entre les extrêmes, entre la vie et le langage, entre le désir d'infinitude et la conscience de la finitude :

Je suis la plaie et le couteau!
Je suis le soufflet et la joue!
Je suis les membres et la roue,
Et la victime et le bourreau !
(BAUDELAIRE, 1975, v. 1, p. 78 )

Cet espace correspond très bien au « petit et monotone » cosmos de l'homme déchu parcouru par le Poète tout au long des Fleurs du mal, voyage dont le bulletin est ainsi résumé dans le dernier poème du recueil :

Pour ne pas oublier la chose capitale,

Nous avons vu partout, et sans l'avoir cherché,

Du haut en bas de l'échelle fatale,

Le spectacle ennuyeux de l'immortel péché

(BAUDELAIRE, 1975, v. 1, p. 132)

Au-delà des Fleurs du mal, les poèmes en prose du Spleen de Paris nous permettent de comprendre que ce petit cosmos est toujours hanté par une dispute entre les forces du chaos et celles de la création, par l'action corrosive de Satan et par la fausse unité divine retrouvée dans l'expérience artistique. Dans sa dernière étude sur Baudelaire, consacrée prioritairement aux dernières années de la production du poète, Antoine Compagnon montre jusqu'à quel point la ville baudelairienne « est perçue du côté de ses implications métaphysiques, voire théologiques, comme une sorte d'archétype ou d'idée reproduisant la multitude éternelle » (COMPAGNON, 2014, p. 172) Les éléments dont se compose cet archétype renvoient toujours à la même source mythique : le récit chrétien de la Création. Sans jamais le citer directement, sauf par l'emploi des mots « chaos » et « tohu-bohu », Baudelaire emprunt à ce mythe biblique les symboles de l'unité, de la fragmentation, de l'ordre et du chaos, qui sont partout dans les poèmes en prose, dont la forme est inspirée des « rapports innombrables » de la ville moderne, selon la célèbre lettredédicace à Arsène Houssaye (BAUDELAIRE, 1975, v. 1, p. 276).

\footnotetext{
${ }^{4}$ Sur la puissance critique de l'oxymore dans la poésie moderne, voir MAULPOIX, 2009, p. 247-250.
} 
On peut finalement retourner à la question du « plein du langage » pour y ajouter qu'elle est inséparable, chez Baudelaire, de la recherche de ce que j'appellerai le plein de l'homme. La découverte de l'autonomie de la langue par rapport à la morale et à la religion n'aboutit pas chez lui sur la quête du néant, mais plutôt sur la mise en œuvre des éléments les plus contradictoires. Coincé entre les « deux postulations », Baudelaire veut montrer que la place du poète se trouve plutôt dans le mouvement à la fois de plongement et de prise de distance par rapport à l'expérience esthétique. Afin de bien comprendre ce mouvement, il nous faut réfléchir sur le paradoxe de l'art baudelairien.

\section{Le paradoxe de l'art}

Dans sa célèbre étude sur Le Spleen de Paris, le critique américain Edward K. Kaplan (2015) propose une lecture du recueil en prose basée sur l'opposition entre poèmes esthétiques et poèmes éthiques. Le livre se composerait d'un enjeu dialectique mis en œuvre grâce au double mouvement d'affirmation et de refus de l'idéalisme esthétique. Ce double mouvement est défini comme un «parcours de découverte de soi » dans lequel presque tous les antagonismes baudelairiens jouent un rôle très important : " Le narrateur de Baudelaire allie regard critique et geste poétique au sein d'un parcours de découverte de soi. C'est un rêveur qui essaie à la fois d'analyser objectivement l'expérience humaine et de compatir avec les individus isolés comme lui » (Kaplan, 2015, p. 20). Le mouvement de retrait qui permet l'établissement du « regard critique » coïncide avec le grand paradoxe de l'art baudelairien. Source d'extase poétique, l'expérience esthétique est pourtant associée à la souffrance, à la perte du moi et souvent à la méchanceté, telle que l'on peut voir par exemple dans la conclusion du " Mauvais vitrier », poème en prose qui peut être lu comme une ironie contre l'absolu esthétique :5 « Mais qu'importe l'éternité de la damnation à qui a trouvé dans une seconde l'infini de la jouissance ?» (BAUDELAIRE, 1975, v. 1, p. 287). La découverte de soi se lie à la prise de conscience de ce « mauvais infini » et de ces limites. ${ }^{6}$ Il est remarquable que le premier poème en

\footnotetext{
${ }^{5}$ C'est bien la lecture qu'en fait Edward Kaplan. Voir KAPLAN, 2015, p. 68.

${ }^{6}$ Dans son analyse du mythe de la chute, Paul Ricœur associe le péché originel à ce qu'il appelle le désir d'infinitude éprouvé par les hommes primordiaux. Le rôle symbolique
} 
prose où la perception des limites de l'expérience esthétique coïncide avec l'élargissement à l'infini de cette même expérience porte dans son titre une référence à la confession catholique. Il s'agit du «Confiteor de l'artiste ", où le poète parle des rapports entre le beau, l'infini et la souffrance : «Que les fins de journées d'automne sont pénétrantes! Ah! pénétrantes jusqu'à la douleur ! car il est de certaines sensations délicieuses dont le vague n'exclut pas l'intensité ; et il n'est pas de pointe plus acérée que celle de l'Infini » (BAUDELAIRE, 1975, v. 1, 278).

Malgré les plusieurs éloges à l'ivresse, Baudelaire s'en prend souvent à la jouissance infinie des sens en y opposant des arguments éthiques voire religieux. C'est bien ce qu'on peut voir dans un article très peu commenté datant de 1852 et intitulé «L'École Païenne », dans lequel Baudelaire dénonce la «folie de l'art», le «goût immodéré de la forme » (BAUDELAIRE, 1976, v. 2, p. 48) des poètes néo-païens. Le seul recours à la matérialité des mots, à l'apparence physique du langage, à la plastique, à la littéralité est condamné par Baudelaire comme une sorte de blasphème, comme la déperdition même de la littérature. Encore une fois, cette condamnation n'est pas sans rapport avec la dualité de la parole baudelairienne et avec l'anthropologie chrétienne à laquelle le poète fait référence dans l'extrait qui suit :

Congédier la passion et la raison, c'est tuer la littérature. Renier les efforts de la société précédente, chrétienne et philosophique, c'est se suicider, c'est refuser la force et les moyens de perfectionnement. S'environner exclusivement des séductions de l'art physique, c'est créer de grandes chances de perdition. Pendant longtemps, bien longtemps, vous ne pourrez voir, aimer, sentir que le beau, rien que le beau. Je prends le mot dans un sens restreint. Le monde ne vous apparaîtra que sous sa forme matérielle. Les ressorts qui le font se mouvoir resteront longtemps cachés (BAUDELAIRE, 1976, v. 2, p. 47).

Baudelaire ne parle pas de l'art en général, mais de la poésie en particulier. Le sujet de son article et la cible de sa critique sont

du Serpent, dans le mythe, serait celui d'offrir à l'homme l'accès à ce «mauvais infini » qui s'oppose à la conscience des limites et, donc, à toute liberté conçue éthiquement. Ce qu'Edward Kaplan appelle l'éthique n'est pas sans rapport avec la reconnaissance de ces limites dans l'action - et dans le langage, j'ajouterais. Voir RICEUR, 2009, p. 467. 
les poètes contemporains ayant adhéré au paganisme pas seulement comme religion mais plutôt comme modèle de création poétique. Il est question d'une critique du langage comme simple apparence et de l'éloge de la parole dans ses rapports avec tout ce qui dépasse le visible. La teneur chrétienne de cette critique est plus qu'évidente. Il n'est pas par hasard que Baudelaire rappelle Saint Augustin dans le cadre de cette dénonciation du « goût immodéré de la forme » :

Je comprends les fureurs des iconoclastes et des musulmans contre les images. J'admets tous les remords de Saint Augustin sur le trop grand plaisir des yeux. Le danger est si grand que j'excuse la suppression de l'objet. La folie de l'art est égale à l'abus de l'esprit (BAUDELAIRE, 1976, v. 2, p. 49).

Voici donc le paradoxe de l'art et, d'une certaine façon, celui de la poésie de Baudelaire : l'autonomie du beau est attachée à la fois à l'extase et à la perdition, au plaisir et au vide, parce que « la spécialisation d'une faculté aboutit au néant » (BAUDELAIRE, 1976, v. 2, p. 49). Pour en finir avec cette question du paradoxe de l'art, il faut ajouter que le rapport à Saint Augustin est moins d'ordre moral que philosophique. Il nous rappelle que la conception baudelairienne du langage s'attache à la dualité de la forme et du sens, semblable à celle du corps et de l'esprit, dont l'origine se trouve dans le mythe de la chute. Baudelaire soutient que l'art et la poésie se tiennent entre ces deux postulats. Entre les apparences et le sens, entre la forme et la philosophie : « la passion frénétique de l'art est un chancre qui dévore le reste ; et, comme l'absence nette du juste et du vrai dans l'art équivaut à l'absence d'art, l'homme entier s'évanouit » (BAUDELAIRE, 1976, v. 2, p. 49).

\section{Le plein de l'homme : poésie, profanation et salut provisoire}

Le paradoxe de l'art s'explique par la quête de cet « homme entier», ambigu et multiforme lui-même. Doué de désir et de conscience, coincé entre l'extase et l'horreur de la vie, cet homme ressemble beaucoup au « monstre incompréhensible » de Pascal, dont la définition se trouve, telle que chez Baudelaire, dans le double mouvement de la célébration lyrique et de l'ironie:

S'il se vante, je l'abaisse

S'il s'abaisse, je le vante

Et le contredis toujours 
Jusques à ce qu'il comprenne

Qu'il est un monstre incompréhensible

(PASCAL, 1991, p. 113).

À la fois théologique et anthropologique, puisqu'ils sont basés sur l'affirmation continuée de la condition déchue de l'homme, les fondements de la poésie baudelairienne ne peuvent pas être cherchés ailleurs que dans le paradoxe de la généralisation du mal, de l'homme déchu comme 1'homme éternel.7 Cette " généralisation de l'enfer de l'homme »(SISCAR, 2010, p. 58) se fait grâce à une radicalisation à laquelle Baudelaire soumet son rapport au mythe de la chute. Détaché de sa reprise typologique dans la figure du Christ, le mythe d'Adam est généralisé à tel point qu'il ne garde plus aucune relation avec une rédemption future. Anthropologique par excellence (RICCEUR, 2009, p. 447), le mythe de la chute est à la fois profané et démythologisé chez Baudelaire grâce à cette radicalisation. Fondement du beau et de la morale baudelairiens, le péché est à la base aussi de la parole poétique, qui est condamnée à ce mouvement éternel de plongement et d'émersion, de systole et de diastole, de « vaporisation et centralisation » (BAUDELAIRE, 1975, v. 1, p. 676).

Baudelaire devient le profanateur du mythe chrétien sans pourtant le tourner en dérision. Tout au contraire, la profanation d'Adam se réalise par le biais de l'affirmation et de la radicalisation, la chute étant la seule forme d'absolu concevable par le poète des Fleurs du mal et du Spleen de Paris. La radicalisation du caractère anthropologique du mythe de la chute originelle le restitue « au libre usage des hommes » (AGAMBEN, 2006 , p. 95) pour offrir au poète une sorte de miroir dans lequel se reflètent la condition humaine en toute sa nudité et aussi celle du poète moderne, condamné à se sacrifier à la méchanceté environnante afin d'y arracher le beau sans pourtant se perdre dans l'inconscience du mal.

Tout cela nous invite à une réévaluation partielle de l'héritage maistrien chez Baudelaire, du moins en ce qui concerne le sacrifice. Alors que pour l'auteur d'Eclaircissements sur le sacrifice il est question plutôt d'un « salut par le sang », chez Baudelaire la rédemption ne paraît jouer plus aucun rôle. Bien que le sacrifice garde toujours chez lui sa valeur sacrée, étant donné que « la peine de Mort est le résultat d'une idée mystique totalement incomprise aujourd'hui » (BAUDELAIRE,

${ }^{7}$ A ce propos, voir COMPAGNON, 1989, p. 105. 
1975, v. 1, p. 683), il faut chercher son véritable sens dans la réflexion baudelairienne sur l'art. Si le mal est partout dans cet homme «si naturellement dépravé » (BAUDELAIRE, 1975, v. 1, p. 665) et dans le monde en général, la poésie ne peut sortir que de la communion avec ce même monde. C'est pourquoi l'art est souvent comparé à l'amour dérivé de la prostitution : «Qu'est-ce que l'art ? Prostitution » (BAUDELAIRE, 1975 , v. 1, p. 649). Comme le poète, « l'amour veut sortir de soi, se confondre avec sa victime, comme le vainqueur avec le vaincu, et cependant conserver des privilèges de conquérant » (BAUDELAIRE, 1975, v. 1, p. 650). En d'autres mots, le sacrifice poétique se réalise paradoxalement en tant que communion avec la victime tout en gardant la différence du poète-bourreau par rapport à elle. On revient encore une fois à la célèbre formule de la « conscience dans le mal » indirectement reprise dans ce fragment de Mon cœur mis à nu consacré au sacrifice : « pour que le sacrifice soit parfait, il faut qu'il y ait assentiment et joie de la part de la victime » (BAUDELAIRE, 1975, v. 1, p. 683). Or, si le poète est défini en même temps comme la victime et le bourreau de soimême, il est à la fois celui qui tue et celui qui meurt, celui qui tombe et celui qui témoigne de sa propre chute. Plongé comme tous les hommes dans le mal généralisé, il est néanmoins le seul capable de marquer sa différence, de retourner à la surface pour respirer et regarder du dehors, que ce soit pendant quelques instants, l'enfer de sa condition humaine à laquelle il est pourtant éternellement condamné. Il me semble que cette différence est moins la prise de conscience de l'illusion romantique et du vide du langage, comme le soutient Barbara Johnson (1979, p. 72), que l'émersion d'une parole po-éthique $e^{8}$ mettant en évidence à la fois notre condition maudite et notre capacité critique. Le rôle de la poésie, du moins chez Baudelaire, est de révéler le " plein de l'homme », ce «monstre incompréhensible » doué de conscience dans son propre mal. Comme Joseph de Maistre, Baudelaire voit dans la vie la matérialisation et la perpétuation du mal. Mas contrairement à son modèle philosophique, il ne voit plus dans le sacrifice du Christ le salut de l'humanité. L'eucharistie catholique, Baudelaire la remplace par la communion poétique, source de volupté et de conscience, une sorte de salut provisoire par la critique dans l'éternité de la chute. Ainsi la parole poétique aurait-elle affaire à ce que Baudelaire appelle « la diminution des traces du péché originel »

\footnotetext{
${ }^{8}$ Sur les rapports entre la différence et la parole éthique/poétique, voir DEGUY, 1987, p. 24.
} 
(BAUDELAIRE, 1975, v. 1, p. 697). Elle serait capable d'ouvrir cet espace intermédiaire entre le " gouffre amer » et la « couvercle du ciel » où se joue toute la dignité de l'homme.

\section{Références}

AGAMBEN, G. Profanations. Trad. Martin Rueff. Paris : Éditions Payot et Rivages, 2006.

BAUDELAIRE, C. Euvres complètes. Texte établi, présenté et annoté par Claude Pichois. Paris : Gallimard, 1975. v. 1. (Coll. Bibliothèque de la Pléiade).

BAUDELAIRE, C. Euvres complètes. Texte établi, présenté et annoté par Claude Pichois. Paris : Gallimard, 1976. v. 2. (Coll. Bibliothèque de la Pléiade).

BONNEFOY, Y. Le Siècle de Baudelaire. Paris : Seuil, 2014.

COMPAGNON, A. Baudelaire devant l'éternel. In : BERCOT, M. ; GUYAUX, A. (Org.). Dix études sur Baudelaire. Paris : Honoré Champion, 1989. p. 71-111.

COMPAGNON, A. Baudelaire : l'irréductible. Paris : Flammarion, 2014.

COMPAGNON, A. Les Antimodernes : de Joseph de Maistre à Roland Barthes. Paris : Gallimard, 2005.

DEGUY, M. La Poésie n'est pas seule : court traité de poétique. Paris : Seuil, 1987.

DE MAISTRE, J. Les Soirées de Saint-Pétersbourg. In : .Euvres. Édition établie par Pierre Glaudes. Paris : Robert Laffont, 2007. p. 401785 .

DUBRAY, J. Pascal et Baudelaire. Paris : Classiques Garnier, 2011.

GUYAUX, A. ; SCEPI, H. (Org.). Lire le Spleen de Paris de Baudelaire. Paris : PUP, 2014.

JOHNSON, B. Défigurations du langage poétique : la seconde révolution baudelairienne. Paris : Flammarion, 1979. 
KAPLAN, E. K. Baudelaire et le Spleen de Paris : l'esthétique, l'éthique et le religieux. Traduction d'Élise Trogrlic. Paris : Classiques Garnier, 2015.

MAULPOIX, J.-M. Pour un lyrisme critique. Paris : José Corti, 2009.

PASCAL, B. Pensées. Paris : Bordas, 1991. Collection Classiques Garnier.

RICCEUR, P. La Symbolique du mal. In : . Le Volontaire et l'involontaire, $I$ : finitude et culpabilité. Paris : Gallimard, 2009. p. 201-577.

SCHELLINO, A. Bibliographie $d u$ Spleen de Paris (1855-2014). Paris : Classiques Garnier, 2015.

SISCAR, M. O grande deserto dea homens. In: . Poesia e crise: ensaios sobre a "crise da poesia" como topos da modernidade. Campinas, SP: Ed. UNICAMP, 2010. 